\title{
Inhibition of Double-Stranded RNA-Induced Inducible Nitric Oxide Synthase Expression by Fraxinellone and Sauchinone in Murine Microglia
}

\author{
Chang Seok Lee, ${ }^{a}$ Cheolhee Won, ${ }^{a}$ Hyouna Yoo, ${ }^{a}$ Eun Hee YI,${ }^{a}$ Yuri Cho,,${ }^{a}$ Jung Woo Maeng, ${ }^{a}$ \\ Sang Hyun SunG, ${ }^{c}$ Sang-Kyu YE, ${ }^{*, a, b}$ and Myung-Hee $\mathrm{CHUNG}^{a}$ \\ ${ }^{a}$ Department of Pharmacology, Seoul National University College of Medicine; and ${ }^{b}$ Ischemic/Hypoxic Disease Institute, \\ Seoul National University College of Medicine; 28 Yongon-Dong, Chongno-Gu, Seoul 110-799, Korea: and ${ }^{c}$ College of \\ Pharmacy, Seoul National University; San 56-1, Sillim-Dong, Gwanak-Gu, Seoul 151-742, Korea. \\ Received May 22, 2009; accepted July 17, 2009
}

\begin{abstract}
Fraxinellone and sauchinone, isolated from natural substance, are known to have an anti-inflammatory effect in inflammatory conditions. However, the anti-inflammatory actions of these compounds have been insufficiently demonstrated in viral-induced neuroinflammation. A viral component (double-stranded (ds)RNA) triggers a toll-like receptor 3-dependent inflammatory response that stimulates pro-inflammatory mediators in the brain. In present study, we initially examined the biological effects of fraxinellone and sauchinone on anti-inflammatory actions in dsRNA-stimulated microglia. Both compounds inhibited dsRNA-induced inducible nitric oxide synthase (iNOS) expression, a major pro-inflammatory enzyme. To demonstrate the mechanism of inhibitory effect on iNOS expression, we further examined the signaling pathway induced by dsRNA in microglia. Our data show that dsRNA promotes the expression of signal transducers and activators of transcription (STAT)1/3 identified as major inflammatory transcription factors as well as activates c-Jun N-terminal kinase (JNK) in an early time. Moreover, both compounds suppressed activation of JNK-STAT1/3 signaling pathway. These results suggest that an anti-inflammatory effect by fraxinellone and sauchinone is mediated via blockade of the JNKSTAT1/3-iNOS signaling pathway in viral-infected microglia.
\end{abstract}

Key words double-stranded RNA; inducible nitric oxide synthase; STAT; c-Jun N-terminal kinase; fraxinellone; sauchinone

Microglia activation is a key factor in defense of the neural parenchyma against infectious diseases, neuroinflammation and neurodegeneration. ${ }^{1)}$ Microglia are characteristically activated in response to neurotropic virus infection. Doublestranded RNA (dsRNA) represents a conserved molecular pattern of virus infection, which accumulates during viral replication and can be sensed by microglia via toll-like receptor 3 (TLR3). ${ }^{2)}$ However, the molecular mechanisms underlying activation of the immunomodulator signaling pathway are not sufficiently characterized in dsRNA-stimulated microglia. A recent report demonstrated that c-Jun N-terminal kinase (JNK) and p38 mitogen-activated protein kinase mediate dsRNA-induced inducible nitric oxide synthase (iNOS) expression in the microglial cell line, BV2. ${ }^{3)}$ Further studies are essential to clarify whether other signaling pathways are involved in iNOS expression by dsRNA.

Signal transducer and activator of transcription (STAT) is a major regulator of inflammatory disease ${ }^{4}$ and a key transcription factor mediating inflammatory responses in brain ischemia. ${ }^{5}$ Previously, we and others reported that activated STAT 1/3 mediate iNOS expression. ${ }^{6-8)}$ We demonstrate in this study that polyinosinic-polycytidylic acid (poly I:C), a synthetic dsRNA, also induces iNOS expression through the activated STAT $1 / 3$ signaling pathway in microglia. Moreover, activated STAT $1 / 3$ are induced via dsRNA-stimulated JNK activation in microglia. Thus, it appears that dsRNA stimulates the JNK-STAT1/3-iNOS signaling pathway in microglia.

We examined the effects of natural compounds displaying neuroprotective activity on JNK-STAT1/3-iNOS inflammatory signaling by dsRNA. Earlier reports show that fraxinellone, the major component of Cortex Dictamni isolated from Chinese medicinal herbs, exerts anti-inflammatory and neu- roprotective effects. ${ }^{9)}$ In addition, sauchinone, a lignin from Saururus chinensis, functions in hepatoprotection and neuroprotection, ${ }^{10,11)}$ and inhibits lipopolysaccharide (LPS)-induced iNOS expression through blockade of nuclear factor (NF)- $\kappa \mathrm{B}$ activation. ${ }^{12,13)}$ However, the signaling mechanisms involved in the anti-inflammatory actions of these compounds are yet to be clearly clarified.

In this study, we show that fraxinellone and sauchinone significantly suppress the protein expression of not only active STAT1/3, but also iNOS and JNK in dsRNA-stimulated BV2 cells. Our data suggest that these compounds promote anti-inflammatory effects through blockade of the JNKSTAT1/3-iNOS signaling pathway in virus-induced neuroinflammatory diseases, and may thus be crucial for developing natural neuroprotective drugs.

\section{MATERIALS AND METHODS}

Materials Polyinosinic-polycytidylic acid (poly I:C) and kinase inhibitors (AG490 and SP600125) were purchased from Sigma (St. Louis, MO, U.S.A.). The concentration of dsRNA employed in all experiments was $25 \mu \mathrm{g} / \mathrm{ml}$. Fraxinellone and sauchinone were donated from Professor Sung. They were isolated and purified from root bark of Dictamnus dasycarpus and herbs of Saururus chinensis, respectively, and identified by spectral analysis, as they previously reported. ${ }^{9,10)}$ The purity of these compounds was determined to be more than $99 \%$ by normalization of the peak areas detected by the high performance liquid chromatography-diode array detector (HPLC-DAD) analysis. Antibodies against inducible NO synthase (iNOS) and alpha-tubulin were purchased from Abcam (Cambridge, U.K.) and Neomarkers Inc. (Fremont, U.S.A.), respectively. JNK antibody was purchased 
(A)

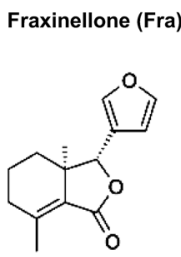

(B)

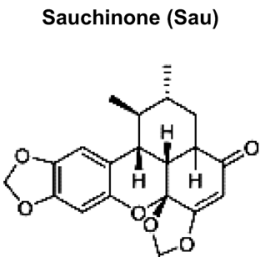

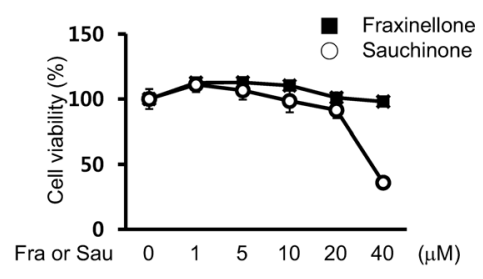

Fig. 1. Cell Viability of Fraxinellone and Sauchinone

Chemical structures of fraxinellone and sauchinone (A). BV2 cells were incubated for $24 \mathrm{~h}$ with the indicated doses of fraxinellone or sauchinone, and cell viability assessed using the MTT assay (B). Data are representative of three independent experiments

from Cell Signaling Technology (MA, U.S.A.). STAT1/3 antibodies were obtained from Santa Cruz Biotechnology (CA, U.S.A.).

Cell Culture BV2-immortalized murine microglial cells were grown in Dulbecco's modified Eagle's medium (DMEM) supplemented with $5 \% \mathrm{FBS}$ at $37^{\circ} \mathrm{C}$ and $5 \% \mathrm{CO}_{2}$.

Western Blot Analysis Western blotting was performed, as described previously. ${ }^{6}$ In brief, cells were washed twice with cold phosphate buffered saline (PBS) and lysed in icecold modified radio immuno precipitation assay (RIPA) buffer (50 mm Tris- $\mathrm{HCl}, \mathrm{pH} 7.4,1 \%$ Nonidet P-40, 0.5\% sodium deoxycholate, $150 \mathrm{~mm} \mathrm{NaCl}$ ) containing protease and phosphatase inhibitors. The lysate was centrifuged and the supernatant collected. Proteins in lysates were separated by SDS-PAGE and transferred to nitrocellulose membrane. The membrane was incubated with primary antibodies and washed, followed by further incubation with peroxidase-conjugated secondary antibodies (Zymed, CA, U.S.A.). Protein bands were visualized using the ECL system (Amersham, Piscataway, NJ, U.S.A.).

MTT Assay Cell viability was assessed by the standard MTT (3-(4,5-dimethylthiazol-2-yl)-2,5-diphenyltrazolium bromide) assay. Cells at the exponential phase were collected and transferred into each well $\left(5 \times 10^{4}\right.$ cells/well $)$. Cells were incubated for $24 \mathrm{~h}$ in the presence of various amounts of fraxinellone or sauchinone (up to $40 \mu \mathrm{M}$ ), and MTT solution added to individual wells. After incubation for $3 \mathrm{~h}$, formazan crystals in each well were dissolved in DMSO, and absorbance read at $540 \mathrm{~nm}$ on a scanning multiwell spectrophotometer (Molecular Device Co., Sunnyvale, CA, U.S.A.). All experiments were performed in triplicate.

Determination of Nitric Oxide (NO) Release BV2 cells were stimulated with dsRNA in the presence or absence of fraxinellone or sauchinone for $24 \mathrm{~h}$. Isolated supernatant fractions were mixed with an equal volume of Griess reagent (Sigma), and incubated at room temperature for $10 \mathrm{~min}$. Nitrite production was determined by measuring optical density at $540 \mathrm{~nm}$.

Reverse Transcription-Polymerase Chain Reaction (RT-PCR) Analysis Total RNA was extracted using TRIzol (Invitrogen Life Technologies) and applied for RT-PCR. cDNA was prepared using a Maxime RT-premix (iNtRON, Seoul, Korea), according to the manufacturer's instructions. PCR was performed with 35 cycles of sequential reactions, specifically, $94^{\circ} \mathrm{C}$ for $1 \mathrm{~min}, 62^{\circ} \mathrm{C}$ for $1 \mathrm{~min}$, and $72^{\circ} \mathrm{C}$ for $1 \mathrm{~min}$. Oligonucleotide primers were purchased from Bioneer (Seoul, Korea). The sequences of PCR primers were as follows: forward, 5'-TTGTCTTCTGCACGAACCTG-3' and re- verse, 5'-CGCAACGCAAGGATTTTATT-3' for TLR3, forward, 5'-CCATGGAGAAGGCTGGGG-3' and reverse, 5' CAAAGTTGTCATGGATGACC-3' for GAPDH.

Statistical Analysis Data were expressed as mean S.E.M. Statistical comparisons were made by Dunnett's multiple comparison test.

\section{RESULTS}

Fraxinellone and Sauchinone Inhibit iNOS Expression and Nitric Oxide (NO) Production in dsRNA-Stimulated Microglia Fraxinellone, a novel degraded limonoid obtained from a methanolic extract of Dictamnus dasycarpus root bark, and sauchinone, a diastereomeric lignin isolated from Saururus chinensis, have neuroprotective and anti-inflammatory effects (Fig. 1A). ${ }^{9-13)}$ We examined the biological effects of these compounds on anti-inflammatory action in dsRNA-stimulated microglia. To assess cytotoxicity, microglia were treated with the two compounds for $24 \mathrm{~h}$, and the level of cell survival was measured using the MTT assay (Fig. 1B). Fraxinellone exerted no cytotoxicity up to $40 \mu \mathrm{M}$, while cytotoxic effects of sauchinone were evident at this concentration. Consequently, the maximum concentration of both compounds employed in all experiments was $20 \mu \mathrm{M}$. Next, we detected the inhibitory effect of fraxinellone and sauchinone on dsRNA-induced iNOS expression, one of the major inflammatory enzymes in microglia. The protein level of iNOS was increased in a time-dependent manner by dsRNA in microglia (Fig. 2A), while fraxinellone and sauchinone significantly inhibited not only iNOS expression (Fig. 2B) but nitric oxide secretion in a dose-dependent manner at the concentration of $0,2,10$ and $20 \mu \mathrm{M}$ (Fig. 2C).

Fraxinellone and Sauchinone Inhibit STAT1/3 Activation in dsRNA-Stimulated Microglia Since dsRNA is a representative inflammatory stimulant and activated STATs enhance inflammatory signaling transduction, we examined whether dsRNA induces STAT1/3 phosphorylation. Phosphotyrosine-STAT1/3 expression was clearly induced in a timedependent manner from about $2 \mathrm{~h}$ after dsRNA treatment in microglia (Fig. 3A). Next, we determined whether dsRNAactivated STAT $1 / 3$ regulate iNOS expression, in view of the finding that LPS-activated STAT1/3 enhanced expression of iNOS, as a target gene. ${ }^{6}$ The treatment with AG490 (a JAKSTAT inhibitor) led to suppression of dsRNA-induced iNOS expression in a dose-dependent manner at the concentration of $0,2,10,20$ and $40 \mu \mathrm{M}$ in microglia (Fig. 2B). Then, we evaluated STAT1/3 activation in the absence or presence of each compound in dsRNA-stimulated microglia (Fig. 3C). 
(A)

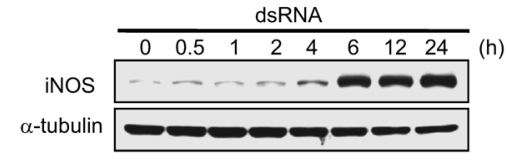

(B)
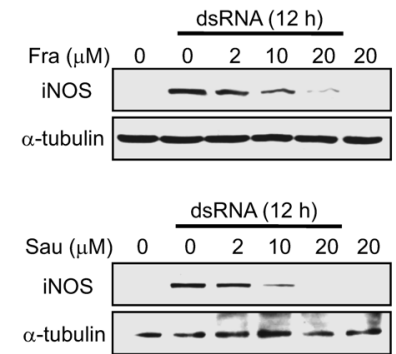

(C)

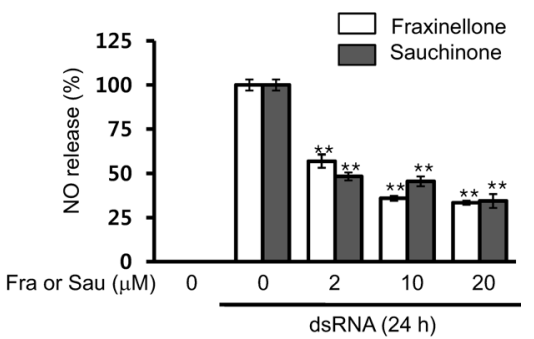

Fig. 2. Inhibition of dsRNA-Induced Inducible Nitric Oxide Synthase (iNOS) and Nitric Oxide (NO) by Fraxinellone and Sauchinone

BV2 cells were incubated for the indicated times with $25 \mu \mathrm{g} / \mathrm{ml}$ dsRNA and iNOS expression was detected by Western blot analysis using antibodies against iNOS and $\alpha$-tubulin (A). BV2 cells were incubated for $12 \mathrm{~h}$ with $25 \mu \mathrm{g} / \mathrm{ml}$ dsRNA following pretreatment for $3 \mathrm{~h}$ with each compound at the concentration of $0,2,10$ and $20 \mu \mathrm{M}$ and iNOS expression was detected by Western blot analysis (B). BV2 cells were pre-treated for $3 \mathrm{~h}$ with each compound at the concentration of $0,2,10$ and $20 \mu \mathrm{M}$ before treatment with $25 \mu \mathrm{g} / \mathrm{ml}$ dsRNA for $24 \mathrm{~h}$. The amount of released NO was determined by measuring nitrite concentrations in media. Data are presented as mean S.E.M. $(* * p<0.01$, comparison with dsRNA-treated cells). Data are representative of three independent experiments (C)

(A)

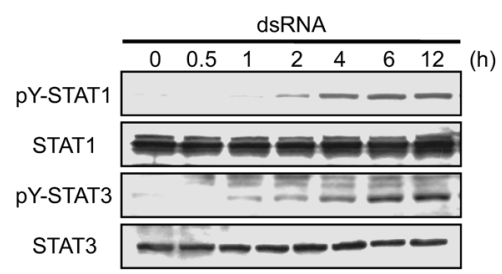

(B)

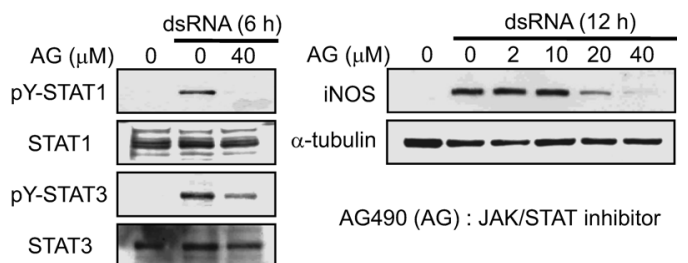

(C)

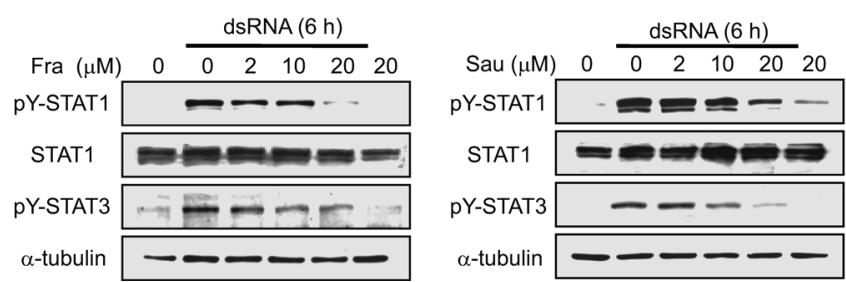

Fig. 3. Inhibition of dsRNA-Induced Phospho-STAT1/3 Expression by Fraxinellone and Sauchinone

BV2 cells were incubated for the indicated times with $25 \mu \mathrm{g} / \mathrm{ml}$ dsRNA. STAT1/3 activation was detected by Western blot analysis using antibodies against phospho-STAT1 (Tyr701), phospho-STAT3 (Tyr705) (A). BV2 cells were incubated for 6 or $12 \mathrm{~h}$ with $25 \mu \mathrm{g} / \mathrm{ml}$ dsRNA following pretreatment for $1 \mathrm{~h}$ with AG490 (JAK/STAT inhibitor) up to a concentration of $40 \mu \mathrm{M}$ (B). BV2 cells were incubated for $6 \mathrm{~h}$ with $25 \mu \mathrm{g} / \mathrm{ml}$ dsRNA following pretreatment for $3 \mathrm{~h}$ with fraxinellone or sauchinone from a minimum concentration of $2 \mu \mathrm{m}$ up to a maximum concentration of $20 \mu \mathrm{m}$. STAT1/3 activation was detected by Western blot analysis as described for Fig. 3A (C).

As expected, both compounds suppressed dsRNA-induced STAT $1 / 3$ activation in a dose-dependent manner in microglia. Our results suggest that activated STAT1/3 induce iNOS expression in dsRNA-stimulated microglia, and finally both fraxinellone and sauchinone effectively are likely to act as modulators of inflammatory disease in the brain.

Fraxinellone and Sauchinone Suppress Activated JNK Which Induces STAT1/3 Activation and iNOS Expression in dsRNA-Stimulated Microglia To identify the upstream signaling molecules regulating dsRNA-activated STAT1/3 expression, we initially examined JNK activation in dsRNAstimulated microglia. In our experiments, JNK activation was significantly enhanced within $0.5 \mathrm{~h}$ in dsRNA-treated microglia (Fig. 4A). Interestingly, the activation time of JNK was distinct from that of STAT1/3 by dsRNA. We propose that $\mathrm{JNK}$ activation at a relatively early time-point mediates STAT1/3 activation and iNOS expression at a later timepoint. Experiments using SP600125 (a JNK inhibitor) confirmed that dsRNA-induced iNOS expression and STAT1/3 activation were markedly suppressed by SP600125 at the concentration of $0,2,10,20$ and $40 \mu \mathrm{M}$ (Figs. 4B, C), while
SB202190 (a p38 inhibitor) and PD98059 (an ERK inhibitor) suppressed iNOS expression to a lower extent (data not shown). Furthermore, fraxinellone and sauchinone suppressed JNK activation in a dose-dependent manner (Fig. 4D). These findings indicate that both compounds inhibit the dsRNA-activated JNK-STAT1/3-iNOS signaling pathway as an inflammatory mechanism in microglia.

Fraxinellone and Sauchinone Inhibit TLR3 Transcription in dsRNA-Stimulated Microglia In addition, we determined the effects of these compounds on TLR3 transcription because TLR3 is a major primary signaling molecule in dsRNA stimulation. ${ }^{2)}$ TLR3 transcripts were increased by dsRNA (Fig. 5A), then, both fraxinellone and sauchinone at the concentration of 10 and $20 \mu \mathrm{M}$ suppressed dsRNA-induced TLR3 transcription in microglia (Fig. 5B). Thus, our data collectively demonstrate that fraxinellone and sauchinone exert anti-inflammatory effects through blockade of JNK-STAT1/3-iNOS-NO as well as the TLR3 signaling pathway in dsRNA-stimulated microglia. 
(A)

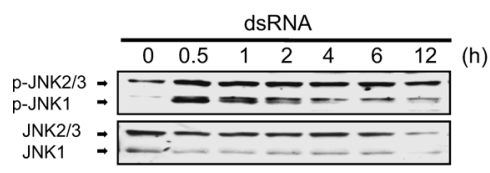

(C)

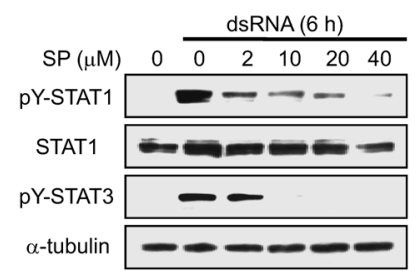

(B)

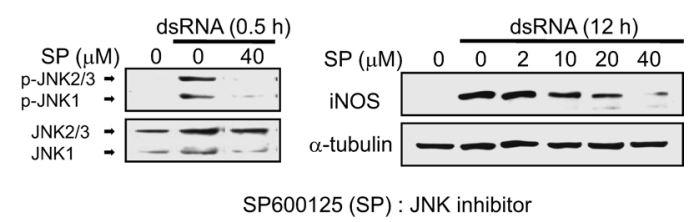

(D)

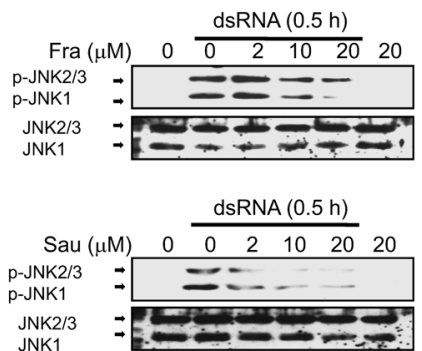

Fig. 4. Inhibition of dsRNA-Induced Phospho-JNK Expression by Fraxinellone and Sauchinone

BV2 cells were incubated for the indicated times with $25 \mu \mathrm{g} / \mathrm{ml}$ dsRNA, and JNK activation was detected by Western blot analysis using an antibody against phospho-JNK (Thr183/Tyr185) (A). BV2 cells were incubated for 0.5 or $12 \mathrm{~h}$ (B) and $6 \mathrm{~h}$ (C) with $25 \mu \mathrm{g} / \mathrm{ml}$ dsRNA following pretreatment for $1 \mathrm{~h}$ with SP600125 (JNK inhibitor) up to a maximum concentration of $40 \mu \mathrm{m}$. Activated STAT1/3 was detected by Western blot analysis as described for Fig. 3. BV2 cells were incubated for $0.5 \mathrm{~h}$ with $25 \mu \mathrm{g} / \mathrm{ml}$ dsRNA following pretreatment for $3 \mathrm{~h}$ with each compound at the concentration of $0,2,10$ and $20 \mu \mathrm{m}$, and activated JNK was detected by Western blot analysis (D).

(A)
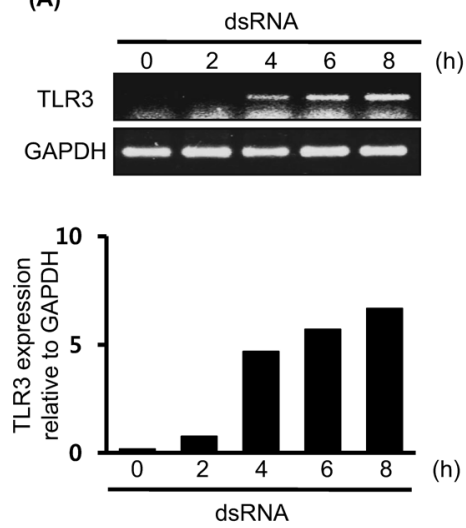
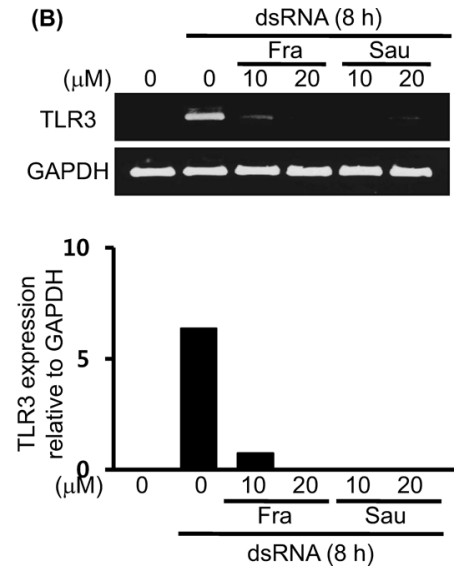

Fig. 5. Inhibition of dsRNA-Induced TLR3 mRNA Expression by Fraxinellone and Sauchinone

BV2 cells were incubated for the indicated times with $25 \mu \mathrm{g} / \mathrm{ml}$ dsRNA (A) or incubated for $8 \mathrm{~h}$ with $25 \mu \mathrm{g} / \mathrm{ml}$ dsRNA in the absence or presence of the compounds (B). Total RNA was isolated, and RT-PCR analysis was performed using the primers described in Materials and Methods. Transcript levels were normalized against those of GAPDH. TLR3 bands were quantified and normalized versus GAPDH bands using ImageJ version $1.35 \mathrm{~d}$ (National Institute of Health Imaging software).

\section{DISCUSSION}

Recent reports show that dsRNA is recognized by TLR3, initiating downstream signaling that leads to expression of inflammatory molecules, such as iNOS and cyclooxygenase2 (COX-2) and cytokines. ${ }^{14,15)}$ However, the mechanism by which dsRNA activates the intracellular signaling pathway and induces expression of inflammatory mediators, particularly in microglia, remains to be established.

As we and others have reported, STAT $1 / 3$ triggers iNOS expression in microglia activated by stimulants, such as LPS, interferon- $\gamma$ or thrombin. ${ }^{6,16,17)}$ However, limited information is available on mediation of pro-inflammatory proteins, such as iNOS, in brain cells by dsRNA-activated STAT. In this report, we initially demonstrate that STAT $1 / 3$ inactivation leads to decreased iNOS expression in dsRNA-stimulated microglia. We propose that dsRNA-activated STAT1/3 are induced by JNK signaling activation. Previously, Kong et al. reported that activated JNK promote dsRNA-induced iNOS expression in microglia. ${ }^{3)}$ Consistent with this finding, we confirmed that JNK inactivation led to decreased iNOS expression in dsRNA-treated microglia. Moreover, our data indicate that dsRNA-activated JNK mediates STAT1/3 activation. There are reports that activated JNK induced interferon $\beta$, as a JAK-STAT activator, which is produced rapidly when dsRNA interacts with TLR3 ${ }^{19,20)}$ In this point, we suspect that dsRNA-activated JNK induces STAT1/3 activation via autocrine interferon $\beta$ production in microglia. Based on the collective results, we propose that dsRNA induces activation of the JNK-STAT1/3-iNOS signaling pathway in microglia.

Next, we tested anti-inflammatory abilities of fraxinellone and sauchinone, which exert neuroprotective effects, in dsRNA-stimulated microglia. While numerous compounds from Chinese medicinal herbs appear to play an immunomodulatory role, limited scientific information is available on the underlying signaling pathways. Here, we focus on 
the inhibitory effects of these compounds on dsRNA-induced JNK and STAT1/3 activation, including iNOS expression. As expected, both compounds inhibited JNK-STAT1/3 activation, iNOS expression and nitric oxide secretion in dsRNAstimulated microglia. In addition, dsRNA-induced COX-2 expression in microglia was suppressed (data not shown).

TLR3 acts as an initiator of dsRNA-induced signaling transduction, and its transcriptional activity is mediated by STAT sites in its proximal promoter. ${ }^{18)}$ The two compounds inhibited the increase of dsRNA-induced TLR3 transcripts in microglia. Accordingly, we propose that STAT1/3 inactivation by fraxinellone and sauchinone is likely to regulate TLR3 transcription and the downstream signaling pathway. Taken together, these compounds may be used to develop effective anti-inflammatory drugs against neuroinflammatory diseases, such as viral-infected encephalitis.

Acknowledgement This study was supported by Grant no. 04-08-072-0 from the SNUH Research Fund.

\section{REFERENCES}

1) Kreutzberg G. W., Trends Neurosci., 19, 312-318 (1996).

2) Town T., Jeng D., Alexopoulou L., Tan J., Flavell R. A., J. Immunol., 176, 3804-3812 (2006).

3) Kong P. J., Lee H. J., Lee S. H., Kim S. Y., Lee S. N., Chun W. J., Kim S. S., Neurosci. Lett., 433, 215-218 (2008).

4) O’Shea J. J., Murray P. J., Immunity, 28, $477-487$ (2008).
5) Planas A. M., Gorina R., Chamorro A., Biochem. Soc. Trans., 34 1267-1270 (2006)

6) Kim H. S., Ye S. K., Cho I. H., Jung J. E., Kim D. H., Choi S., Kim Y S., Park C. G., Kim T. Y., Lee J. W., Chung M. H., Free Radic. Biol. Med., 41, 1392-1403 (2006).

7) de Prati A. C., Ciampa A. R., Cavalieri E., Zaffini R., Darra E., Menegazzi M., Suzuki H., Mariotto S., Curr. Med. Chem., 12, 18191828 (2005).

8) Kleinert H., Schwarz P. M., Förstermann U., Biol. Chem., 384, 13431364 (2003).

9) Yoon J. S., Sung S. H., Kim Y. C., J. Nat. Prod., 71, 208-211 (2008).

10) Sung S. H., Kim Y. C., J. Nat. Prod., 63, 1019-1021 (2000).

11) Song H., Kim Y. C., Moon A., Biol. Pharm. Bull., 26, 1428-1430 (2003).

12) Hwang B. Y., Lee J. H., Jung H. S., Kim K. S., Nam J. B., Hong Y. S., Paik S. G., Lee J. J., Planta Med., 69, 1096-1101 (2003).

13) Lee A. K., Sung S. H., Kim Y. C., Kim S. G., Br. J. Pharmacol., 139, $11-20$ (2003).

14) Nakamichi K., Saiki M., Kitani H., Kuboyama Y., Morimoto K., Takayama-Ito M., Kurane I., Neurosci. Lett., 13, 222-227 (2007).

15) Mogensen T. H., Paludan S. R., Microbiol. Mol. Biol. Rev., 65, 131150 (2001).

16) Huang C., Ma R., Sun S., Wei G., Fang Y., Liu R., Li G., J. Neuroimmunol., 204, 118-125 (2008).

17) Chen J. C., Ho F. M., Pei-Dawn Lee Chao., Chen C. P., Jeng K. C., Hsu H. B., Lee S. T., Wen Tung Wu., Lin W. W., Eur. J. Pharmacol., 521, 9-20 (2005).

18) Liu P., Jamaluddin M., Li K., Garofalo R. P., Casola A., Brasier A. R., J. Virol., 81, 1401-1411 (2007).

19) Malmgaard L., J. Interferon Cytokine Res., 24, 439-454 (2004)

20) Peters K. L., Smith H. L., Stark G. R., Sen G. C., Proc. Natl. Acad. Sci. U.S.A., 99, 6322-6327 (2002). 